\title{
A LEMMA ON A FREE GROUP
}

\author{
AKIHIKO MORIMOTO
}

The purpose of this note is to prove the following proposition, which is made use of in the preceding paper by Mr. M. Kuranishi in this number. First we shall explain the notations. Let $f(X, Y)=X^{k_{1}} Y^{l_{1}} \ldots X^{k_{s}} Y^{l_{s}}$ be a word with respect to two letters, and let $G$ be a free group generated by two free generators $x$ and $y$. Next we define "。"-composition in $G$ by $a \circ b=f(a, b)$. Now the proposition which we want to prove is the following

PROPOSITION. If the following two conditions

$$
\begin{aligned}
& (x \circ y) \circ y=x \circ(y \circ y) \\
& (x \circ x) \circ y=x \circ(x \circ y)
\end{aligned}
$$

hold, then the word $f$ is one of the following five forms

$$
1, X, Y, X Y, Y X \text {. }
$$

Proof. It is easy to see that $(y \circ x) \circ x=y \circ(x \circ x)$ and $(y \circ y) \circ x$ $=y \circ(y \circ x)$ must be satisfied. So we can assume $k_{1} \neq 0$. We can see that it is sufficient to prove that $s$ is not greater than one. First we assume that $f$ $=X^{k_{1}} Y^{l_{1}} \ldots X^{k_{s}}$, where $s \geqslant 2$ and all exponents of $X$ and $Y$ are different from zero. Then we can easily see that the condition (1) is a non-trivial relation between $x$ and $y$, which is a contradiction. Next we assume that $f=X^{k_{1}} Y^{l_{1}}$ $\ldots X^{k_{s}} Y^{l_{s}}$. From condition (1) we get the following relation

$$
\begin{aligned}
& \left(x^{k_{1}} y^{l_{1}} \ldots x^{k_{s}} y^{l_{s}}\right)^{k_{1}} y^{l_{1}} \ldots\left(x^{k_{1}} y^{l_{1}} \ldots x^{k_{s}} y^{l_{s}}\right)^{k_{s}} y^{l_{s}} \\
& \quad=x^{k_{1}} y^{\left(\Sigma k_{i}+\Sigma l_{i}\right) l_{1}} \ldots x^{k_{s}} y^{\left(\Sigma k_{i}+\Sigma l_{i}\right) l_{s}},
\end{aligned}
$$

from which we can conclude that $s$ must be even and

$$
k_{s-i+1}+k_{i}=0 \text { for } i=1, \ldots, s \text { must hold. }
$$

From (2) we get in the same manner the equalities

Received April 26, 1954. 


$$
l_{s-i+1}+l_{i}=0 \text { for } i=1, \ldots, s .
$$

So we can write as $f=X^{k_{1}} Y^{l_{1}} \ldots X^{k_{t}} Y^{l_{t}} X^{-k_{t}} Y^{-l_{t}} \ldots X^{-k_{1}} Y^{-l_{1}}$. Then from (2) we can conclude that $t \geq 2$ and successively $k_{1}=k_{2}, k_{2}=k_{3}, \ldots, k_{t-1}=k_{t}$ and from (1) $l_{1}=l_{2}, l_{2}=l_{3}, \ldots, l_{t-1}=l_{t}$. And again from (1) we get a non-trivial relation between $x$ and $y$, and complete our proof. 